\title{
Radical Reduction of Aromatic Azides to Amines with Tributylgermanium Hydride
}

Luisa Benati, Giorgio Bencivenni, Rino Leardini, * Matteo Minozzi, Daniele Nanni, Rosanna Scialpi,

Piero Spagnolo, * and Giuseppe Zanardi

\author{
Dipartimento di Chimica Organica "A. Mangini", Università di Bologna, \\ Viale Risorgimento 4, I-40136 Bologna, Italy
}

spagnolo@ms.fci.unibo.it

\section{Supporting information}

\section{Table of contents:}

Page S2-S3. Experimental procedures.

Pages S4-S8. Analytical and spectral data for new compounds 3a-d, 4f-h, 5f-h and 6i-m, o. 
General Procedure for the Preparation of Azides 1a-q. The already known aryl azides 1a-q were prepared by diazotization of the corresponding anilines $\mathbf{2 a - q}$, all commercially available (Aldrich) except biphenyl-2,2'-diamine (2k), ${ }^{1}$ followed by treatment with sodium azide according to a standard procedure. $^{2}$ All the azides were characterized on the basis of physical and/or spectral data. $\left(\mathbf{1 a}{ }^{3} \mathbf{1 b}{ }^{4}\right.$ $\left.\mathbf{1 c},{ }^{5} \mathbf{1 d},{ }^{6} 1 \mathrm{e},{ }^{7} \mathbf{1 f},{ }^{7} \mathbf{1 g},{ }^{8} \mathbf{1 h},{ }^{5} 1 \mathbf{i},{ }^{9} \mathbf{1 j},{ }^{4} \mathbf{1 k},{ }^{10} 1 \mathbf{1},{ }^{11} \mathbf{1 m},{ }^{7} \mathbf{1 n},{ }^{12} \mathbf{1 o},{ }^{13} \mathbf{1 p},{ }^{14} \mathbf{1 q}{ }^{15}\right)$.<smiles>[R]c1ccc([N])cc1</smiles>

1a: $\mathrm{R}=\mathrm{Cl}$

1b: $R=O M e$

1c: $\mathrm{R}=\mathrm{COMe}$

1d: $\mathrm{R}=\mathrm{CN}$

1e: $\mathrm{R}=\mathrm{NO}_{2}$<smiles>[R]c1ccc(N)cc1</smiles>

2a: $\mathrm{R}=\mathrm{Cl}$

2b: $\mathrm{R}=\mathrm{OMe}$

2c: $\mathrm{R}=\mathrm{COMe}$

2d: $\mathrm{R}=\mathrm{CN}$

2e: $\mathrm{R}=\mathrm{NO}_{2}$<smiles>[R]c1cccc([N])c1</smiles><smiles>[R]c1ccccc1N</smiles>

1i: $R=M e$

1j: $\mathrm{R}=\mathrm{OMe}$

1k: $\mathrm{R}=2-\mathrm{NH}_{2} \mathrm{C}_{6} \mathrm{H}_{4}$

1I: $R=F$

10: $\mathrm{R}=\mathrm{COMe}$

1p: $\mathrm{R}=\mathrm{Br}$

1q: $R=1$<smiles>[R]c1ccccc1N</smiles><smiles>Nc1cccc2ccccc12</smiles>

2f: $\mathrm{R}=\mathrm{Cl}$

2g: $\mathrm{R}=\mathrm{OMe}$

2i: $\mathrm{R}=\mathrm{Me}$

$2 \mathrm{~m}: \mathrm{R}=\mathrm{Cl}$

2n

2j: $\mathrm{R}=\mathrm{OMe}$

2o: $\mathrm{R}=\mathrm{COMe}$

2h: $\mathrm{R}=\mathrm{COMe}$

2k: $\mathrm{R}=2-\mathrm{NH}_{2} \mathrm{C}_{6} \mathrm{H}_{4}$

2p: $\mathrm{R}=\mathrm{Br}$

2I: $R=F$

2q: $R=$ I<smiles>Nc1cccc2ccccc12</smiles>

1n<smiles>[R]c1cccc(N)c1</smiles>

${ }^{1}$ Ross, S. D.; Kahan, G. J.; Leach, W. A. J. Am. Chem. Soc, 1952, 74, 4122.

${ }^{2}$ Smith, P.A.S.; Brown, B.B. J. Am. Chem. Soc. 1951, 73, 2438.

${ }^{3}$ Butler, R. N.; Collier, S.; Fleming, A. F. M. J. Chem. Soc. Perkin Trans. 2 1996, 801.

${ }^{4}$ Huber, M. L.; Pinhey, J. T. J. Chem. Soc. Perkin Trans. 1 1990, 721.

${ }_{6}^{5}$ Ohba, Y.; Kubo, S.; Nakai, M.; Nagai, A.; Yoshimoto, M. Bull. Chem. Soc. Jpn. 1986, 59, 2317.

${ }^{6}$ Nicolaides, A.; Enyo, T.; Miura, D.; Tomioka, H. J. Am. Chem. Soc. 2001,123, 2628.

${ }^{7}$ Lieber E.; Ramachandra Rao, C. N.; Chao T. S., Hoffman C. W. W. Anal. Chem. 1957, 29, 916.

${ }^{8}$ Nunno, L. Di; Scilimati, A. Tetrahedron 1986, 42, 3913.

${ }^{9}$ Morawietz, J.; Sander, W.; Traeubel, M. J. Org. Chem. 1995, 60; 6368.

${ }^{10}$ Murata, S.; Tsuji, H.; Tomioka, H. Bull. Chem. Soc. Jpn. 1994, 67, 895.

${ }^{11}$ Leyva, E.; Munoz, D.; Platz, M. S. J. Org. Chem. 1989, 54, 5938.

${ }^{12}$ Klump, S. P.; Shechter, H. Tetrahedron Lett. 2002, 438421.

${ }^{13}$ Takada, K.; Kan-Woon, T.; Boulton, A. J. J. Org. Chem. 1982, 47, 4323.

${ }^{14}$ Avemaria, F.; Zimmermann, V.; Braese, S. Syn. Lett. 2004, 7, 1163.

${ }^{15}$ Tomioka, H.; Sawai, S. Org. Biomol. Chem. 2003, 1, 4441. 
General Procedure for the Reactions of Azides 1a-q with Tributylgermanium Hydride. A toluene $(15 \mathrm{~mL})$ solution containing the appropriate azide 1 (1 mmol), tributylgermanium hydride $(1.1 \mathrm{mmol})$ and benzenethiol $(0.1 \mathrm{mmol})$ was refluxed for ca $30 \mathrm{~min}$, after which time TLC normally showed the virtual disappearance of the starting substrate. In the case of the azides 1e, $1 \mathbf{p}$ and $1 \mathbf{q}$ prolongation of the reaction time up to $60 \mathrm{~min}$ did not cause any significant change in the ensuing products. After cooling to room temperature, the solvent was removed in vacuo and the residue subjected to chromatographic separation on silica gel by progressive elution with light petroleum/diethyl ether mixtures. Yields of the isolated products are collected in Table 1. The anilines 2 and 3-methyl-2,1benzisoxazole 7 (Barth, A.; Corrie, J. E. T.; Gradwell, M. J.; Maeda, Y.; Mäntele, W.; Meier, T.; Trentham, D. R. J. Am. Chem. Soc. 1997, 119, 4149) were identified by spectral comparison with authentic samples. Analytical and spectral data of the unknown germylated anilines 3a-d, 4f-h, 5f-h and $\mathbf{6 i - m , ~} \mathbf{0}$ were as follows. 


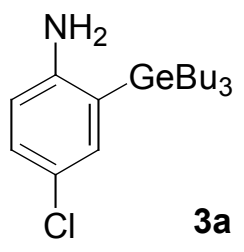

4-Chloro-2-tributylgermylaniline (3a). oil; IR $v_{\max }\left(\mathrm{cm}^{-1}\right) 3459$ and 3380 $\left(\mathrm{NH}_{2}\right) ;{ }^{1} \mathrm{H}$ NMR $(400 \mathrm{MHz}) \delta$ 0.87-0.90 (9 H, m), 1.00-1.05 (6 H, m), 1.33-1.37 $(12 \mathrm{H}, \mathrm{m}), 3.66\left(2 \mathrm{H}, \mathrm{br} \mathrm{s}, \mathrm{NH}_{2}\right), 6.55(1 \mathrm{H}, \mathrm{d}, J=8.4 \mathrm{~Hz}), 7.06\left(1 \mathrm{H}, \mathrm{dd}, J_{l}=\right.$ $\left.8.4 \mathrm{~Hz}, J_{2}=2.6 \mathrm{~Hz}\right), 7.09(1 \mathrm{H}, \mathrm{d}, J=2.6 \mathrm{~Hz}),{ }^{13} \mathrm{C} \mathrm{NMR}(100 \mathrm{MHz}) \delta 12.8$ $\left(\mathrm{CH}_{2}\right), 13.7\left(\mathrm{CH}_{3}\right), 26.4\left(\mathrm{CH}_{2}\right), 27.3\left(\mathrm{CH}_{2}\right), 116.2(\mathrm{CH}), 123.4(\mathrm{C}), 125.7(\mathrm{C})$, $129.2(\mathrm{CH}), 134.4(\mathrm{CH}), 149.4(\mathrm{C})$; MS (ESI) (rel inten) $372[(\mathrm{M}+6)-1]^{-}(38), 370[(\mathrm{M}+4)-1]^{-}$ (100), $368[(\mathrm{M}+2)-1]^{-}(61), 366(\mathrm{M}-1)^{-}$(36). Anal. calcd for $\mathrm{C}_{18} \mathrm{H}_{32} \mathrm{ClGeN}$ : C, 58.35; H, 8.71; N, 3.78. Found: C, 58.46; H, 8.70; N, 3.79 .

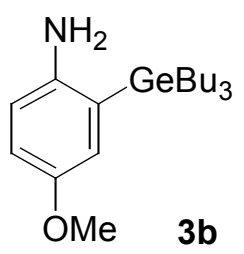

4-Methoxy-2-tributylgermylaniline (3b). oil; IR $v_{\max }\left(\mathrm{cm}^{-1}\right) 3448$ and 3368 $\left(\mathrm{NH}_{2}\right)$; ${ }^{1} \mathrm{H}$ NMR (400 MHz) $\delta$ 0.84-0.92 (9 H, m), 0.99-1.07 (6 H, m), 1.25-1.43 $(12 \mathrm{H}, \mathrm{m}), 3.43\left(2 \mathrm{H}, \mathrm{br} \mathrm{s}, \mathrm{NH}_{2}\right), 3.75(3 \mathrm{H}, \mathrm{s}), 6.60(1 \mathrm{H}, \mathrm{d}, J=8.6 \mathrm{~Hz}), 6.71(1$ $\left.\mathrm{H}, \mathrm{dd}, J_{1}=8.6 \mathrm{~Hz}, J_{2}=2.9 \mathrm{~Hz}\right), 6.79(1 \mathrm{H}, \mathrm{d}, J=2.9 \mathrm{~Hz}),{ }^{13} \mathrm{C} \mathrm{NMR}(100 \mathrm{MHz})$ 反 $13.0\left(\mathrm{CH}_{2}\right), 13.7\left(\mathrm{CH}_{3}\right), 26.5\left(\mathrm{CH}_{2}\right), 27.4\left(\mathrm{CH}_{2}\right), 55.6\left(\mathrm{CH}_{3}\right), 114.4(\mathrm{CH}), 116.2$ (CH), $120.9(\mathrm{CH}), 125.6(\mathrm{C}), 144.6(\mathrm{C}), 152.4(\mathrm{C})$; $\mathrm{MS}(\mathrm{EI}) \mathrm{m} / \mathrm{z}$ (rel inten) $367\left(\mathrm{M}^{+}+4,16\right), 365\left(\mathrm{M}^{+}+\right.$ 2, 11), $363\left(\mathrm{M}^{+}, 14\right), 310$ (83), 308 (63), 306 (45), 252 (60), 250 (46), 248 (36), 196 (100), 194 (91), 192 (59), 123 (30). Anal. calcd for $\mathrm{C}_{19} \mathrm{H}_{35} \mathrm{GeNO}$ : C, 62.33; H, 9.64; N, 3.83. Found: C, 62.48; H, 9.66; N, 3.82 .

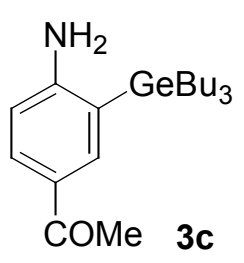

4'-Amino-3'-tributylgermylacetophenone (3c). oil; IR $v_{\max }\left(\mathrm{cm}^{-1}\right) 3489$ and $3366\left(\mathrm{NH}_{2}\right), 1579(\mathrm{CO}) ;{ }^{1} \mathrm{H}$ NMR (400 MHz) $\delta$ 0.86-0.96 (9 H, m), 1.01-1.10 (6 $\mathrm{H}, \mathrm{m}), 1.24-1.48(12 \mathrm{H}, \mathrm{m}), 2.50(3 \mathrm{H}, \mathrm{s}), 4.17\left(2 \mathrm{H}, \mathrm{br} \mathrm{s}, \mathrm{NH}_{2}\right), 6.59(1 \mathrm{H}, \mathrm{d}, J=$ $8.4 \mathrm{~Hz}), 7.77\left(1 \mathrm{H}, \mathrm{dd}, J_{1}=8.4 \mathrm{~Hz}, J_{2}=2.2 \mathrm{~Hz}\right), 6.86(1 \mathrm{H}, \mathrm{d}, J=2.2 \mathrm{~Hz}),{ }^{13} \mathrm{C}$ NMR (100 MHz) $\delta 12.8\left(\mathrm{CH}_{2}\right), 13.7\left(\mathrm{CH}_{3}\right), 17.6\left(\mathrm{CH}_{3}\right), 26.4\left(\mathrm{CH}_{2}\right), 27.3\left(\mathrm{CH}_{2}\right)$, $113.6(\mathrm{CH}), 121.9(\mathrm{C}), 127.6(\mathrm{C}), 131.0(\mathrm{CH}), 136.7(\mathrm{CH}), 155.5$ (C), 196.7 (CO); MS (EI) $\mathrm{m} / z$ (rel inten) $379\left(\mathrm{M}^{+}+4,<1\right), 377\left(\mathrm{M}^{+}+2,<1\right), 375\left(\mathrm{M}^{+},<1\right), 322$ (100), 320 (81), 318 (57), 264 (77), 262 (51), 260 (34), 208 (86), 206 (64), 204 (50), 135 (9). Anal. calcd for $\mathrm{C}_{20} \mathrm{H}_{35} \mathrm{GeNO}$ : C, 63.53; H, 9.64; N, 3.70. Found: C, 63.47; H, 9.63; N, 3.71. 


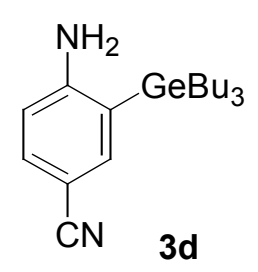

4-Amino-3-tributylgermylbenzonitrile (3d). oil; IR $v_{\max }\left(\mathrm{cm}^{-1}\right) 3494$ and 3371 $\left(\mathrm{NH}_{2}\right), 2214(\mathrm{CN}) ;{ }^{1} \mathrm{H}$ NMR $(300 \mathrm{MHz}) \delta$ 0.79-0.96 (9 H, m), 0.98-1.09 $(6 \mathrm{H}$, m), 1.20-1.48 (12 H, m), $4.16\left(2 \mathrm{H}, \mathrm{br} \mathrm{s}, \mathrm{NH}_{2}\right), 6.6(1 \mathrm{H}, \mathrm{d}, J=8.2 \mathrm{~Hz}), 7.38(1$ $\left.\mathrm{H}, \mathrm{dd}, J_{1}=8.2 \mathrm{~Hz}, J_{2}=1.9 \mathrm{~Hz}\right), 7.42(1 \mathrm{H}, \mathrm{d}, J=1.9 \mathrm{~Hz}),{ }^{13} \mathrm{C}$ NMR $(75.45$ MHz) $\delta 12.7\left(\mathrm{CH}_{2}\right), 13.7\left(\mathrm{CH}_{3}\right), 26.3\left(\mathrm{CH}_{2}\right), 27.2\left(\mathrm{CH}_{2}\right), 114.1(\mathrm{CH}), 117.8$ (CN), $120.7(\mathrm{C}), 123.5(\mathrm{C}), 133.7(\mathrm{CH}), 139.6(\mathrm{CH}), 154.5(\mathrm{C})$; MS (EI) $\mathrm{m} / z$ (rel inten) $305\left[\left(\mathrm{M}^{+}+4\right)\right.$ 57), 54)], 303 [( $\left.\left.\left.\left.\mathrm{M}^{+}+2\right)-57\right), 47\right)\right], 301$ [( $\mathrm{M}^{+}$- 57), 31)], 247 (47), 245 (42), 243 (18), 191 (100), 189 (67), 187 (42), 118 (45). Anal. calcd for $\mathrm{C}_{19} \mathrm{H}_{32} \mathrm{GeN}_{2}$ : C, 63.20; H, 8.93; N, 7.76. Found: C, 63.39; H, $8.92 ; \mathrm{N}, 7.77$.

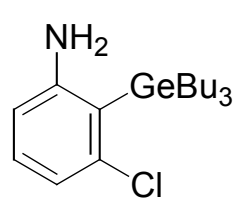

4f

3-Chloro-2-tributylgermylaniline (4f). oil; IR $v_{\max }\left(\mathrm{cm}^{-1}\right) 3462$ and 3392 $\left(\mathrm{NH}_{2}\right) ;{ }^{1} \mathrm{H}$ NMR (400 MHz) $\delta$ 0.81-0.95 (9 H, m), 1.11-1.24 (6 H, m), 1.28-1.47 $(12 \mathrm{H}, \mathrm{m}), 3.89\left(2 \mathrm{H}, \mathrm{br} \mathrm{s}, \mathrm{NH}_{2}\right), 6.46\left(1 \mathrm{H}, \mathrm{dd}, J_{l}=7.9 \mathrm{~Hz}, J_{2}=1.1 \mathrm{~Hz}\right), 6.72(1$ $\left.\mathrm{H}, \mathrm{dd}, J_{1}=7.9 \mathrm{~Hz}, J_{2}=1.1 \mathrm{~Hz}\right), 6.99(1 \mathrm{H}, \mathrm{t}, J=7.9 \mathrm{~Hz}),{ }^{13} \mathrm{C} \mathrm{NMR}(100 \mathrm{MHz}) \delta$ $13.71\left(\mathrm{CH}_{2}\right), 15.7\left(\mathrm{CH}_{3}\right), 26.4\left(\mathrm{CH}_{2}\right), 27.5\left(\mathrm{CH}_{2}\right), 113.6(\mathrm{CH}), 119.8(\mathrm{CH}), 121.8$ (C), $130.2(\mathrm{CH}), 141.9(\mathrm{C}), 153.2(\mathrm{C})$; MS (EI) $\mathrm{m} / z$ (rel inten) $316\left[\left(\mathrm{M}^{+}+6\right)-57,43\right], 314\left[\left(\mathrm{M}^{+}+4\right)-\right.$ 57, 100], 312 [( $\left.\left.\mathrm{M}^{+}+2\right)-57,87\right], 310\left(\mathrm{M}^{+}-57,47\right), 258$ (42), 256 (49), 254 (35), 252 (18), 202 (43), 200 (48), 198 (27), 196 (16), 166 (63), 164 (73), 162 (51), 160 (14), 129 (23), 127 (59). Anal. calcd for $\mathrm{C}_{18} \mathrm{H}_{32} \mathrm{ClGeN}$ : C, 58.35; H, 8.71; N, 3.78. Found: C, 58.47; H, 8.69; N, 3.77.

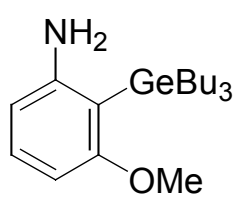

$4 \mathrm{~g}$

3-Methoxy-2-tributylgermylaniline (4g). oil; IR $v_{\max }\left(\mathrm{cm}^{-1}\right) 3485$ and 3387 $\left(\mathrm{NH}_{2}\right) ;{ }^{1} \mathrm{H}$ NMR $(400 \mathrm{MHz}) \delta$ 0.86-0.94 (9 H, m), 1.01-1.08 (6 H, m), 1.25-1.46 $(12 \mathrm{H}, \mathrm{m}), 3.71(3 \mathrm{H}, \mathrm{s}), 3.78\left(2 \mathrm{H}, \mathrm{br} \mathrm{s}, \mathrm{NH}_{2}\right), 6.24\left(2 \mathrm{H}, \mathrm{dq}, J_{d}=8.0 \mathrm{~Hz}, J_{q}=\right.$ $1.0 \mathrm{~Hz}), 7.06(1 \mathrm{H}, \mathrm{t}, J=8.0 \mathrm{~Hz})$; due to the very small isolated amounts the ${ }^{13} \mathrm{C}$ NMR spectrum was not recorded; MS (EI) $m / z$ (rel inten) $367\left(\mathrm{M}^{+}+4,3\right), 365$ ( $\mathrm{M}^{+}+$2, 2), 363 ( $\left.\mathrm{M}^{+}, 2\right), 310$ (100), 308 (75), 306 (61), 252 (82), 250 (49), 248 (42), 196 (69), 194 (54), 192 (35), 166 (48), 164 (42), 162 (29), 123 (27). Anal. calcd for $\mathrm{C}_{19} \mathrm{H}_{35} \mathrm{GeNO}$ : C, 62.33; H, 9.64; N, 3.83. Found: C, 62.56; H, 9.62; N, 3.84. 


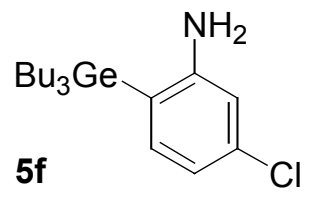

5-Chloro-2-tributylgermylaniline (5f). oil; IR $v_{\max }\left(\mathrm{cm}^{-1}\right) 3465$ and 3345 $\left(\mathrm{NH}_{2}\right) ;{ }^{1} \mathrm{H}$ NMR $(400 \mathrm{MHz}) \delta$ 0.81-0.92 (9 H, m), 0.97-1.05 (6 H, m), 1.26$1.43(12 \mathrm{H}, \mathrm{m}), 3.74(2 \mathrm{H}, \text { br s, NH})_{2}, 6.61(1 \mathrm{H}, \mathrm{d}, J=2.0 \mathrm{~Hz}), 6.71(1 \mathrm{H}$, dd, $\left.J_{1}=7.9 \mathrm{~Hz}, J_{2}=2.0 \mathrm{~Hz}\right), 7.08(1 \mathrm{H}, \mathrm{d}, J=7.9 \mathrm{~Hz}),{ }^{13} \mathrm{C} \mathrm{NMR}(100 \mathrm{MHz})$

$\delta 12.9\left(\mathrm{CH}_{2}\right), 13.7\left(\mathrm{CH}_{3}\right), 26.4\left(\mathrm{CH}_{2}\right), 27.3\left(\mathrm{CH}_{2}\right), 114.6(\mathrm{CH}), 118.3(\mathrm{CH}), 121.3(\mathrm{C}), 136.2(\mathrm{CH})$, 139.2 (C), 152.1 (C); MS (EI) $m / z$ (rel inten) $316\left[\left(\mathrm{M}^{+}+6\right)-57,33\right], 314\left[\left(\mathrm{M}^{+}+4\right)-57,79\right], 312$ [( $\left.\left.\mathrm{M}^{+}+2\right)-57,49\right], 310\left(\mathrm{M}^{+}-57,32\right), 258$ (27), 256 (59), 254 (42), 252 (20), 202 (58), 200 (100), 198 (73), 196 (43), 129 (10), 127 (23). Anal. calcd for $\mathrm{C}_{18} \mathrm{H}_{32} \mathrm{ClGeN}$ : C, 58.35; H, 8.71; N, 3.78. Found: $\mathrm{C}$, $58.57 ; \mathrm{H}, 8.70 ; \mathrm{N}, 3.76$.

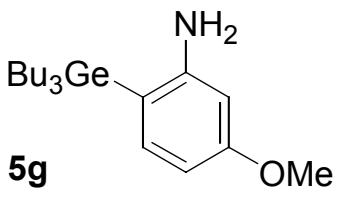

5-Methoxy-2-tributylgermylaniline (5g). oil; IR $v_{\max }\left(\mathrm{cm}^{-1}\right) 3425$ and $3375\left(\mathrm{NH}_{2}\right) ;{ }^{1} \mathrm{H}$ NMR $(400 \mathrm{MHz}) \delta$ 0.84-0.92 $(9 \mathrm{H}, \mathrm{m}), 0.96-1.02(6 \mathrm{H}, \mathrm{m})$, 1.28-1.42 (12 H, m), $3.69\left(2 \mathrm{H}\right.$, br s, $\left.\mathrm{NH}_{2}\right), 3.76(3 \mathrm{H}, \mathrm{s}), 6.19(1 \mathrm{H}, \mathrm{d}, J=$ $2.3 \mathrm{~Hz}), 6.34\left(1 \mathrm{H}, \mathrm{dd}, J_{I}=8.2 \mathrm{~Hz}, J_{2}=2.3 \mathrm{~Hz}\right), 7.08(1 \mathrm{H}, \mathrm{d}, J=8.2 \mathrm{~Hz})$; due to the very small isolated amounts the ${ }^{13} \mathrm{C}$ NMR spectrum was not recorded; MS (EI) $\mathrm{m} / \mathrm{z}$ (rel inten) $367\left(\mathrm{M}^{+}+4,3\right), 365\left(\mathrm{M}^{+}+2,2\right), 363\left(\mathrm{M}^{+}, 1\right), 310(64), 308$ (61), 306 (49), 252 (62), 250 (45), 248 (28), 196 (100), 194 (75), 192 (56), 123 (29). Anal. calcd for $\mathrm{C}_{19} \mathrm{H}_{35} \mathrm{GeNO}$ : C, 62.33; H, 9.64; N, 3.83. Found: C, 62.47; H, 9.62; N, 3.84.

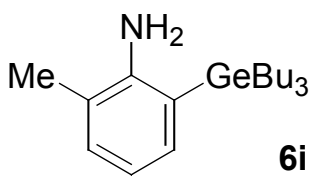

6-Methyl-2-tributylgermylaniline (6i). oil; IR $v_{\max }\left(\mathrm{cm}^{-1}\right) 3481$ and 3398 $\left(\mathrm{NH}_{2}\right) ;{ }^{1} \mathrm{H}$ NMR $(300 \mathrm{MHz}) \delta 0.88(9 \mathrm{H}, \mathrm{t}, J=7.1 \mathrm{~Hz}), 0.98-1.08(6 \mathrm{H}, \mathrm{m})$, 1.25-1.45 (12 H, m), $2.16(3 \mathrm{H}, \mathrm{s}), 3.67\left(2 \mathrm{H}, \mathrm{br} \mathrm{s}, \mathrm{NH}_{2}\right), 6.70(1 \mathrm{H}, \mathrm{t}, J=$ 7.40 Hz), 7.00-7.12 (2 H, m), ${ }^{13} \mathrm{C}$ NMR $(75.45 \mathrm{MHz}) \delta 13.1\left(\mathrm{CH}_{2}\right), 13.7$ $\left(\mathrm{CH}_{3}\right), 18.0\left(\mathrm{CH}_{3}\right), 26.5\left(\mathrm{CH}_{2}\right), 27.4\left(\mathrm{CH}_{2}\right), 118.2(\mathrm{CH}), 121.5(\mathrm{C}), 122.6(\mathrm{C}), 130.9(\mathrm{CH}), 133.0(\mathrm{CH})$, 149.0 (C); MS (EI) $m / z$ (rel inten) $351\left(\mathrm{M}^{+}+4,7\right), 349\left(\mathrm{M}^{+}+2,5\right), 347\left(\mathrm{M}^{+}, 3\right), 294$ (79), 292 (66), 290 (50), 236 (44), 234 (34), 232 (20), 180 (100), 178 (85), 176 (58), 106 (29). Anal. calcd for $\mathrm{C}_{19} \mathrm{H}_{35} \mathrm{GeN}$ : C, 65.18; H, 10.08; N, 4.00. Found: C, 65.37; H, 10.06; N, 3.99.

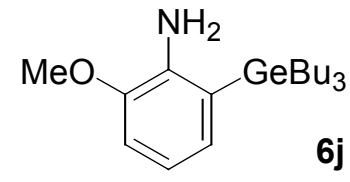

6-Methoxy-2-tributylgermylaniline (6j). oil; IR $v_{\max }\left(\mathrm{cm}^{-1}\right) 3477$ and $3387\left(\mathrm{NH}_{2}\right) ;{ }^{1} \mathrm{H}$ NMR $(300 \mathrm{MHz}) \delta 0.88(9 \mathrm{H}, \mathrm{t}, J=7.1 \mathrm{~Hz}), 0.97-1.10(6$ $\mathrm{H}, \mathrm{m}), 1.25-1.44(12 \mathrm{H}, \mathrm{m}), 3.84(3 \mathrm{H}, \mathrm{s}), 3.89\left(2 \mathrm{H}, \mathrm{br} \mathrm{s}, \mathrm{NH}_{2}\right), 6.69-6.80$ 
$(2 \mathrm{H}, \mathrm{m}), 6.82\left(1 \mathrm{H}, \mathrm{dd}, J_{1}=7.0 \mathrm{~Hz}, J_{2}=1.9 \mathrm{~Hz}\right) ;{ }^{13} \mathrm{C} \mathrm{NMR}(75.45 \mathrm{MHz}) \delta 13.0\left(\mathrm{CH}_{2}\right), 13.7\left(\mathrm{CH}_{3}\right)$, $26.5\left(\mathrm{CH}_{2}\right), 27.4\left(\mathrm{CH}_{2}\right), 55.3\left(\mathrm{CH}_{3}\right), 110.4(\mathrm{CH}), 117.9(\mathrm{CH}), 123.2(\mathrm{C}), 126.7(\mathrm{CH}), 140.4(\mathrm{C}), 146.7$ (C); MS (EI) $m / z$ (rel inten) $367\left(\mathrm{M}^{+}+4,14\right), 365\left(\mathrm{M}^{+}+2,9\right), 363\left(\mathrm{M}^{+}, 8\right), 310(100), 308$ (75), 306 (61), 252 (66), 250 (50), 248 (44), 196 (98), 194 (77), 192 (52), 123 (14). Anal. calcd for $\mathrm{C}_{19} \mathrm{H}_{35} \mathrm{GeNO}$ C, 62.33; H, 9.64; N, 3.83. Found: C, 62.56; H, 9.61; N, 3.84.

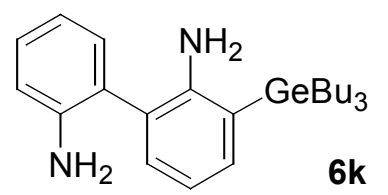

3-Tributylgermyl[1,1'-biphenyl]-2,2'-diamine (6k). oil; IR $v_{\max }\left(\mathrm{cm}^{-1}\right)$ 3469 and $3377\left(\mathrm{NH}_{2}\right) ;{ }^{1} \mathrm{H}$ NMR $(400 \mathrm{MHz}) \delta$ 0.78-0.97 $(9 \mathrm{H}, \mathrm{t}, J=7.00$ $\mathrm{Hz}), 0.99-1.11(6 \mathrm{H}, \mathrm{m}), 1.23-1.47(12 \mathrm{H}, \mathrm{m}), 6.78\left(1 \mathrm{H}, \mathrm{dd}, J_{l}=8.0 \mathrm{~Hz}\right.$, $\left.J_{2}=1.1 \mathrm{~Hz}\right), 6.78\left(1 \mathrm{H}, \mathrm{dd}, J_{1}=7.3 \mathrm{~Hz}, J_{2}=1.7 \mathrm{~Hz}\right), 6.80-6.86(2 \mathrm{H}, \mathrm{m})$, $7.09\left(1 \mathrm{H}, \mathrm{dd}, J_{1}=7.3 \mathrm{~Hz}, J_{2}=1.7 \mathrm{~Hz}\right), 7.11-7.20(2 \mathrm{H}, \mathrm{m}), 7.23\left(1 \mathrm{H}, \mathrm{dd}, J_{1}=7.3 \mathrm{~Hz}, J_{2}=1.7 \mathrm{~Hz}\right),{ }^{13} \mathrm{C}$ NMR $(100 \mathrm{MHz}) \delta 13.1\left(\mathrm{CH}_{2}\right), 13.7\left(\mathrm{CH}_{3}\right), 26.5\left(\mathrm{CH}_{2}\right), 27.4\left(\mathrm{CH}_{2}\right), 115.5(\mathrm{CH}), 118.4(\mathrm{CH}), 118.8$ (CH), $123.6(\mathrm{C}), 124.0(\mathrm{C}), 125.1(\mathrm{C}), 128.7(\mathrm{CH}), 131.1(\mathrm{CH}), 131.4(\mathrm{CH}), 134.8(\mathrm{CH}), 144.1(\mathrm{C})$, $148.4(\mathrm{C}) ; \mathrm{MS}(\mathrm{ESI})\left(\right.$ rel inten) $451[(\mathrm{M}+4)+\mathrm{Na}]^{+}(25), 449[(\mathrm{M}+2)+\mathrm{Na}]^{+}(15), 447(\mathrm{M}+\mathrm{Na})^{+}$ $(11), 429[(\mathrm{M}+4)+1]^{+}(100), 427[(\mathrm{M}+2)+1]^{+}(79), 425(\mathrm{M}+1)^{+}(54), 371(23), 369(18), 367$ (13), 245 (21), 243 (16), 241 (12), 189 (52), 187 (36), 185 (33). Anal. calcd for $\mathrm{C}_{24} \mathrm{H}_{38} \mathrm{GeN}_{2}$ : C, 67.48; H, 8.97; N, 6.56. Found: C, 67.62; H, 8.96; N, 6.54.

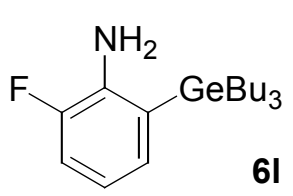

6-Fluoro-2-tributylgermylaniline (6l). oil; IR $v_{\max }\left(\mathrm{cm}^{-1}\right) 3482$ and 3396 $\left(\mathrm{NH}_{2}\right) ;{ }^{1} \mathrm{H}$ NMR (400 MHz) $\delta$ 0.82-0.94 (9 H, m), 0.9-1.10 (6 H, m), 1.26$1.44(12 \mathrm{H}, \mathrm{m}), 3.76\left(2 \mathrm{H}\right.$, br s, $\left.\mathrm{NH}_{2}\right), 6.68\left(1 \mathrm{H}, \mathrm{ddd}, J_{1}=8.0 \mathrm{~Hz}, J_{2}=7.3 \mathrm{~Hz}\right.$, $\left.J_{3(H-F)}=4.6 \mathrm{~Hz}, \mathrm{H}-4\right), 6.91-6.98(2 \mathrm{H}, \mathrm{m}, \mathrm{H}-3, \mathrm{H}-5) ;{ }^{13} \mathrm{C} \mathrm{NMR}(100 \mathrm{MHz}) \delta$ $13.0\left(\mathrm{CH}_{2}\right), 13.7\left(\mathrm{CH}_{3}\right), 26.5\left(\mathrm{CH}_{2}\right), 27.3\left(\mathrm{CH}_{2}\right), 115.1(\mathrm{CH}, \mathrm{d}, J=19.1 \mathrm{~Hz}), 118.3(\mathrm{CH}, \mathrm{d}, J=6.1 \mathrm{~Hz})$, $126.4(\mathrm{C}, \mathrm{s}), 130.0(\mathrm{CH}, \mathrm{d}, J=4.0 \mathrm{~Hz}), 138.6(\mathrm{C}, \mathrm{d}, J=9.7 \mathrm{~Hz}), 151.6(\mathrm{C}, \mathrm{d}, J=242 \mathrm{~Hz}) . \mathrm{MS}(\mathrm{ESI})$ $\left(\right.$ rel inten) $378[(\mathrm{M}+4)+\mathrm{Na}]^{+}(35), 376[(\mathrm{M}+2)+\mathrm{Na}]^{+}(29), 374(\mathrm{M}+\mathrm{Na})^{+}(10), 356[(\mathrm{M}+4)+1]^{+}$ (100), $354[(\mathrm{M}+2)+1]^{+}(92), 352(\mathrm{M}+1)^{+}(73)$. Anal. calcd for $\mathrm{C}_{18} \mathrm{H}_{32} \mathrm{FGeN}$ : C, 61.06; H, 9.11; N, 3.96. Found: C, 61.24; H, 9.09; N, 3.97.

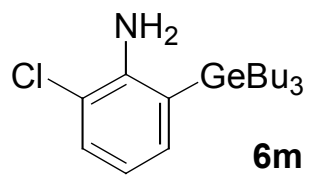

6-Chloro-2-tributylgermylaniline (6m). oil; IR $v_{\max }\left(\mathrm{cm}^{-1}\right) 3486$ and 3393 $\left(\mathrm{NH}_{2}\right) ;{ }^{1} \mathrm{H}$ NMR $(400 \mathrm{MHz}) \delta$ 0.83-0.96 (9 H, m), 0.97-1.10 (6 H, m), 1.22$1.42(12 \mathrm{H}, \mathrm{m}), 4.13\left(2 \mathrm{H}\right.$, br s, $\left.\mathrm{NH}_{2}\right), 6.67(1 \mathrm{H}, \mathrm{t}, J=7.6 \mathrm{~Hz}), 7.08(1 \mathrm{H}, \mathrm{d}$, 
$J=7.8 \mathrm{~Hz}), 7.22(1 \mathrm{H}, \mathrm{d}, J=7.3 \mathrm{~Hz}),{ }^{13} \mathrm{C} \mathrm{NMR}(100 \mathrm{MHz}) \delta 13.0\left(\mathrm{CH}_{2}\right), 13.7\left(\mathrm{CH}_{3}\right), 26.4\left(\mathrm{CH}_{2}\right), 27.2$ $\left(\mathrm{CH}_{2}\right), 118.7(\mathrm{CH}), 119.4(\mathrm{C}), 124.9(\mathrm{C}), 129.5(\mathrm{CH}), 133.4(\mathrm{CH}), 139.1(\mathrm{C}), 146.6(\mathrm{C})$; MS (EI) m/z (rel inten) $373\left(\mathrm{M}^{+}+6,<1\right), 371\left(\mathrm{M}^{+}+4,<1\right), 369\left(\mathrm{M}^{+},<1\right), 316$ (39), 314 (87), 312 (72), 310 (44), 258 (43), 256 (63), 254 (43), 252 (28), 202 (47), 200 (100), 198 (61), 196 (44), 166 (9), 164 (33), 162 (25), 160 (18), 129 (7), 127 (14). Anal. calcd for $\mathrm{C}_{18} \mathrm{H}_{32} \mathrm{ClGeN}$ : C, 58.35; H, 8.71; N, 3.78. Found: C, $58.40 ; \mathrm{H}, 8.70 ; \mathrm{N}, 3.77$.

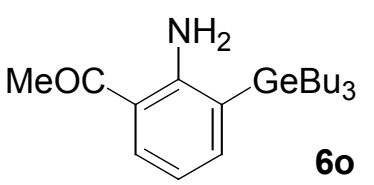

2'-Amino-3'-tributylgermylacetophenone (6o). oil; IR $v_{\max }\left(\mathrm{cm}^{-1}\right) 3496$ and $3317\left(\mathrm{NH}_{2}\right) ;{ }^{1} \mathrm{H}$ NMR $(400 \mathrm{MHz}) \delta 0.88(9 \mathrm{H}, \mathrm{t}, J=7.1 \mathrm{~Hz}), 1.00$ $1.09(6 \mathrm{H}, \mathrm{m}), 1.28-1.42(12 \mathrm{H}, \mathrm{m}), 2.58(3 \mathrm{H}, \mathrm{s}), 6.56\left(2 \mathrm{H}, \mathrm{br} \mathrm{s}, \mathrm{NH}_{2}\right)$, $6.64\left(1 \mathrm{H}, \mathrm{dd}, J_{1}=8.1 \mathrm{~Hz}, J_{2}=7.0 \mathrm{~Hz}\right), 7.32\left(1 \mathrm{H}, \mathrm{dd}, J_{1}=7.0 \mathrm{~Hz}, J_{2}=\right.$

$1.5 \mathrm{~Hz}), 7.72\left(1 \mathrm{H}, \mathrm{dd}, J_{1}=8.1 \mathrm{~Hz}, J_{2},=1.5 \mathrm{~Hz}\right),{ }^{13} \mathrm{C} \mathrm{NMR}(100 \mathrm{MHz}) \delta 13.0\left(\mathrm{CH}_{2}\right), 13.7\left(\mathrm{CH}_{3}\right), 26.4$ $\left(\mathrm{CH}_{2}\right), 27.3\left(\mathrm{CH}_{2}\right), 28.1\left(\mathrm{CH}_{3}\right), 115.5(\mathrm{CH}), 117.2(\mathrm{C}), 125.7(\mathrm{C}), 132.7(\mathrm{CH}), 140.9(\mathrm{CH}), 154.5(\mathrm{C})$, 201.3 (CO). MS (EI) $m / z$ (rel inten) $379\left(\mathrm{M}^{+}+4,10\right), 377\left(\mathrm{M}^{+}+2,4\right), 375\left(\mathrm{M}^{+}, 4\right), 322(100), 320$ (79), 318 (61), 264 (41), 262 (32), 260 (29), 208 (68), 206 (56), 204 (40), 135 (7). Anal. calcd for $\mathrm{C}_{20} \mathrm{H}_{35} \mathrm{GeNO}$ : C, 63.53; H, 9.33; N, 3.70. Found: C, 63.68; H, 9.30; N, 3.71 .

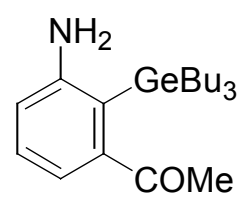

4h

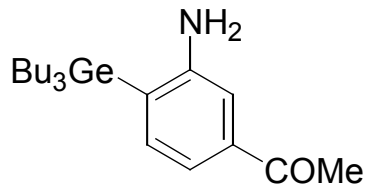

$5 h$

The germylanilines $\mathbf{4 h}$ and $\mathbf{5 h}$ were obtained in very small amounts as an unresolved oily mixture and were characterized by GC-MS analysis (starting $\mathrm{T}$ : $80{ }^{\circ} \mathrm{C}$ [3']; rate: $15{ }^{\circ} \mathrm{C} / \mathrm{min}$; final $\mathrm{T}$ : $270{ }^{\circ} \mathrm{C}$ ). The identification was based on spectral analogies with other germyl compounds. $1^{\text {st }}$ isomer $\left(22.3^{\prime}\right)$ : MS (EI) $m / z$ (rel inten) $322\left[\left(\mathrm{M}^{+}+4\right)-57,84\right], 320\left[\left(\mathrm{M}^{+}\right.\right.$ $+2)-57,70], 318\left(\mathrm{M}^{+}-57,48\right], 208(48), 206(33), 204(25), 135(47) .2^{\text {nd }}$ isomer $\left(24.5^{\prime}\right): 322\left[\left(\mathrm{M}^{+}+\right.\right.$ 4) - 57, 48], $320\left[\left(\mathrm{M}^{+}+2\right)-57,43\right], 318\left(\mathrm{M}^{+}-57,33\right], 264$ (31), 262 (26), 260 (16), 208 (66), 206 (49), 204 (31), 135 (16). Anal. calcd for $\mathrm{C}_{20} \mathrm{H}_{35} \mathrm{GeNO}$ (mixture of isomers): C, 63.53; H, 9.33; N, 3.70. Found: C, 63.37; H, 9.31; N, 3.71. 